\title{
CIENCIA\&SALUD
}

\section{Obesidad: Mecanismos, fisiopatología y tratamiento integral}

desde un enfoque educativo.

Obesity: mechanisms, pathophysiology and comprenhensive

\section{treatment.}

Lina Marĺa Martinez Sanchez ', Sara Pérez².

1 Docente Titular e Investigadora Universidad Pontifica Bolivariana, Medellín Colombia

2 Estudiante de medicina Universidad Pontifica Bolivariana, Medellín Colombia.

Contactos: sara.pereza@upb.edu.colinam.martinez@upb.edu.co

\section{RESUMEN}

\section{Cómo citar:}

Martinez Sanchez, L. M., \& Pérez, S. Obesidad: Mecanismos, fisiopatología y tratamiento integral. Revista Ciencia Y Salud, 6(1), Pág. 71-80. https:// doi.org/10.34192/

cienciaysalud.v6i1.395

Recibido: 16/nov/2021

Aceptado: 10/feb/2022

Publicado: 14/Feb/2022

\section{c) (†)()}

En la actualidad, la obesidad constituye un problema de salud pública al presentarse como una enfermedad metabólica adversa y prevalente, que al encontrarse en un ambiente nocivo donde la comida es barata, sabrosa, ultra procesada y abundante; aumenta a un ritmo acelerado. Aproximadamente el 17\% de los niños estadounidenses y $35 \%$ de los adultos son obesos y los gastos anuales atribuidos a la obesidad y cuidados relacionados superan los 190.000 millones de dólares, constituyendo así un problema para los sistemas de salud y los recursos destinados a estos, es por esto que la promoción y la prevención cobran importancia, al igual que los tratamientos integrales, en los que se tienen en cuenta todas las esferas del individuo y la naturaleza multifactorial de esta enfermedad, es por esto que el objetivo de la presente revisión es plantear una revisión de los mecanismos, fisiopatología y tratamientos que existen actualmente para el manejo integral de la obesidad, con la finalidad de establecer sus ventajas, desventajas y resaltar su importancia, sobre todo desde el punto de vista de la promoción, prevención en salud y salud mental. Para la realización de este artículo, se llevó a cabo una búsqueda bibliográfica en bases de datos como WoS, Education-Research, Lilacs y Redalyc, mediante la selección de palabras clave como "obesidad", "sobrepeso" "fisiopatología" y "tratamiento". Tras la identificación de los artículos de interés, se llevó a cabo una lectura crítica para seleccionar los que mejor se correlacionaran con el objetivo de la revisión, delimitando así una muestra final.

\section{Palabras Clave: Obesidad, Sobrepeso, Fisiopatología, Epigenómica, Psicoterapia}

\section{ABSTRACT}

In the 21st century, obesity constitutes a public health problem as it presents itself as an adverse and prevalent metabolic disease, rather than being in a toxic environment; in which the food is cheap, tasty, ultra processed and abundant; increases at an accelerated rate. Approximately $17 \%$ of American children and $35 \%$ of adults are obese, and the annual expenditures attributed to obesity and related care exceed $\$ 190$ billion, thus constituting a problem for health systems and the resources allocated to them. This is why promotion and prevention become important, as well as comprehensive 
treatments, in which all spheres of the individual and the multifactorial nature of this disease are taken into account, that is why the objective of this review is propose a review of the mechanisms, pathophysiology and treatments that currently exist for the comprehensive management of obesity, in order to establish its advantages, disadvantages and highlight its importance. To carry out this, a bibliographic search was carried out in databases such as WoS, Education-Research, Lilacs and Redalyc, by selecting keywords such as "obesity", "overweight" "pathophysiology" "treatment". After identifying the articles of interest, a critical reading was carried out to select those that best correlated with the objective of the review, thus delimiting a final sample.

\section{Keywords: Obesity, Physiopathology, Epigenomics, Psychotherapy, Overweight}

\section{INTRODUCCIÓN}

La obesidad es una enfermedad metabólica adversa y prevalente que aumenta a un ritmo que no puede ser explicado solo por factores genéticos; los factores ambientales juegan un papel protagónico en su aparición, lo que explica la intervención de interacciones genéticas/epigenéticas y otros factores fisiopatológicos.1,2,3

La obesidad es considerada la pandemia del siglo XXI, con reportes de prevalencia que aumentan cada año. En 2016, más de 1.900 millones de adultos tenían sobrepeso y 650 millones eran obesos.4,5 Aproximadamente el $17 \%$ de los niños estadounidenses y el $35 \%$ de los adultos son obesos y los gastos anuales atribuibles a la obesidad y los cuidados relacionados superan los 190000 millones de dólares.3,6,7

Los estudios de asociación solo explican parte de la variación genética en el riesgo de la enfermedad, lo que sugiere que se deben tener en cuenta más factores para comprender que la obesidad es multifactorial y multicausal, con entidades diferentes en cada individuo5

La epigenética está asociada a cambios en el material genético como la metilación del ácido desoxirribonucleico (ADN) que se produce en las citosinas adyacentes a guaninas denominados islas CpG; otro cambio es la acetilación y/o metilación postraduccional (adición de grupos metilo o acetilos al ADN) de las histonas; y los microARN, que son secuencias cortas de ácido ribonucleico (ARN) que interfieren con la producción de proteínas.1,2,8,9,10,11,12

La exposición ambiental de un individuo refleja un estado epigenético que se adquiere como respuesta a factores como la nutrición, hábitos de vida y ambientales, que pueden estar relacionados con riesgo de enfermedad en la vejez.1,2,5,9,13,14,15

La exposición intrauterina a condiciones adversas y factores ambientales posnatales también pueden contribuir al riesgo de obesidad por modificaciones epigenéticas9.

La exposición a una edad temprana a disruptores endocrinos obesogénicos se ha relacionado con efectos latentes sobre la obesidad.12,16 Las exposiciones ambientales pueden inducir alteraciones persistentes en el epigenoma, que pueden posteriormente conducir en un aumento de la adipogénesis, adiposidad y peso corporal, incluso a lo largo de varias generaciones.16

Los biomarcadores epigenéticos que se traducen del tejido diana a la sangre pueden tener relevancia clínica, ya que pueden analizarse fácilmente.1,3 Estos deben tener una capacidad predictiva que proporcione mejores resultados que los actuales métodos de diagnóstico y terapéuticos.1,2 
Tabla 1. Contribuyentes de obesidad2,3

\begin{tabular}{|c|c|c|}
\hline \multirow{5}{*}{ Obesidad } & Microorganismos (infectobesidad) & $\begin{array}{c}\text { Efectos climáticos que afectan } \\
\text { gasto energético }\end{array}$ \\
\hline & Fecundidad entre padres obesos & $\begin{array}{c}\text { Alteraciones en la homeostasis } \\
\text { del apetito }\end{array}$ \\
\hline & $\begin{array}{c}\text { Aumento de la edad para el } \\
\text { primer embarazo }\end{array}$ & $\begin{array}{c}\text { Efectos farmacológicos no } \\
\text { deseados }\end{array}$ \\
\hline & $\begin{array}{c}\text { Apareamiento entre personas } \\
\text { con sobrepeso }\end{array}$ & Consumo excesivo de alimentos \\
\hline & $\begin{array}{l}\text { Exposición a contaminantes } \\
\text { ambientales }\end{array}$ & Cambios en el epigenoma \\
\hline
\end{tabular}

Los primeros marcadores epigenéticos para la obesidad que se pueden detectar al nacer han sido identificados e incluyen el receptor $X$ retinoico y la metilación del promotor en tejido de cordón umbilical.12,16

\section{MATERIALES Y MÉTODOS}

Para la realización de este artículo se realizó una búsqueda bibliográfica en bases de datos como WoS, Education-Research, Lilacs y Redalyc, mediante la selección de palabras clave como "obesidad", "sobrepeso", "fisiopatología", "tratamiento", "epigenetica" "terapia”, entre otras. Tras la identificación de los artículos de interés, se llevó a cabo una lectura crítica de los títulos, el resumen, las palabras clave y el desarrollo de los textos, para seleccionar los que mejor se correlacionaran con el objetivo de la revisión, delimitando así una muestra final significativa.

\section{Tratamiento integral}

Tanto el apetito, como el peso corporal son regulados por un sistema neuroendocrino que compromete al intestino y al cerebro, los cuales se encuentran altamente integrados. Este sistema, evolutivamente, fue diseñado para rastrear cambios a corto y largo plazo de la ingesta y el gasto energético, con el objetivo de garantizar la homeostasis energética, el peso corporal y defenderse de la escasez de alimentos, por lo cual tiene la capacidad de almacenar la energía como grasa corporal y reducir hasta en un $20 \%$ la tasa metabólica o la energía gastada durante la actividad, lo que antiguamente era fundamental para la supervivencia.2,17

Actualmente, esta regulación neuroendocrina juega en contra del estilo de vida convencional, debido a lo que muchos autores han etiquetado como un ambiente alimentario tóxico, en el que la comida es ahora barata, abundante, ultra procesada, sabrosa, desproporcionada, con infinitas combinaciones de azúcar, grasa, sal y una alta carga calórica. Otro aspecto determinante es la reducción del gasto de energía en las actividades cotidianas, esto debido principalmente, a la digitalización, lo que ha aumentado drásticamente el sedentarismo. Por otro lado, la relación cultural con la comida y el entendimiento individual que se tiene sobre consumo de alimentos, resulta ser de gran importancia a la hora de determinar la aparición de esta patología, por lo que debe considerarse a la hora de determinar un tratamiento efectivo 2,17

El sistema neuroendocrino que defiende contra los peligros de la escasez y pérdida de peso no es igualmente hábil para responder a la abundancia calórica o a un medio ambiente con gasto energético reducido, en el cual la pérdida de calorías no es un requisito para comer. Además, tampoco distingue con facilidad los esfuerzos intencionales para perder peso, por lo cual los individuos deben fortalecer cada vez más un control riguroso en su índice de masa corporal con esfuerzos cognitivos y conductuales para regular la ingesta de alimentos y actividad física.2,17

A continuación, se mencionarán modificaciones en el estilo de vida que propician la pérdida de peso, pero debe aclararse que para que estas sean efectivas y sostenibles en el tiempo, el paciente debe recibir un acompañamiento y educación constantes, con el fin de que pueda adaptarse a un nuevo estilo de vida. 


\section{CIENCIA\&SALUD}

\section{Dieta:}

La restricción calórica es esencial para reducir la pérdida de peso clínicamente significativa. Se recomienda mantener un déficit calórico de entre $500-750 \mathrm{kcal} /$ día, con una pérdida media resultante de 0,5 a 0,75 kg por semana. Las guías estadounidenses recomiendan que aproximadamente $15-20 \%$ de las calorías diarias se deriven de las proteínas, mientras que el $20-35 \%$ de las grasas, con no más de $10 \%$ de saturadas, y el resto de los carbohidratos, particularmente frutos secos, cereales y verduras. Reducir el tamaño de las porciones, el exceso de azúcar y grasa, son formas convenientes de lograr el déficit deseado. Es por esto que se recomienda generalmente una dieta de 1200 - $1500 \mathrm{kcal} /$ día para individuos que pesen menos de 113 kilogramos y 1500 - $1800 \mathrm{kcal} /$ día para quienes tengan un peso mayor. Nunca se recomiendan dietas de 200 a $800 \mathrm{kcal} /$ día a menos que haya una necesidad clínica de rápida pérdida de peso.17,18,19

A pesar de lo mencionado anteriormente, es importante mencionar que en los últimos 15 años se ha visto que la distribución de macronutrientes per se no es tan significativa para lograr una pérdida de peso, siempre y cuando se respete el déficit calórico, por lo cual, la dieta elegida no resulta tan significativa (mediterránea, alta en proteínas, de bajo consumo de carbohidratos, ayuno intermintente), puesto que diversos estudios que comparaban dietas en las que prevalecía la disminución de consumo de carbohidratos con otras bajas en grasas, demostraron una pérdida de peso similar, siempre y cuando se mantuviera el déficit, para lo cual es indispensable acompañar al paciente y ayudarlo a entender su relación con la comida, para que pueda cambiar su estilo de vida a uno que beneficie su estado de salud y se mantenga con el tiempo.17

La composición de macronutrientes en la dieta, sin embargo, puede modificar el riesgo cardiometabólico, y respecto a esto se ha encontrado que una dieta de índice glucémico bajo reduce la hemoglobina A1c, en comparación con una dieta baja en grasas, a pesar de que la pérdida de peso fue similar, por lo tanto, los factores de riesgo cardiometabólico deben tenerse en cuenta al elegir la dieta a seguir y se deben tener en cuenta las condiciones y comorbilidades de cada paciente.17

Otro aspecto importante a tener en cuenta es la pérdida de adherencia, por lo cual las dietas altamente hipocalóricas pueden resultar negativas al existir un efecto rebote. La revisión sistémica de Franz, et. al. demostró que la dieta VLCD (ingesta de $<800 \mathrm{kcal} /$ día) a corto plazo resulta superior a la LCD (ingesta 800 $1600 \mathrm{kcal} /$ día), con una pérdida de peso de 17,9kg (16\%) a los seis meses, después de lo cual sus beneficios comienzan a disminuir por diversas razones, entre ellas, el efecto rebote relacionado con la pérdida de la adherencia por la alta restricción calórica. Por otro lado, el reemplazo de comidas ya sea parcial o total, con sustitutos bajos en calorías, ofrece un método fácil y conveniente para la restricción de ingesta calórica, este, según un metaanálisis realizado por Heymsfield, et. at. produce una pérdida de peso mayor a los 3 meses y 1 año, con menor tasa de deserción y mejoría de las condiciones patológicas asociadas a la obesidad como diabetes mellitus tipo 2, por lo que se recomienda sobre las altamente hipocalóricas, ya que representa mayores beneficios para quienes las acogen. 18,20,21

\section{Deporte:}

Los programas de modificación de estilo de vida recomiendan 150-180 minutos por semana de actividad aerobia moderada como, caminar a peso ligero o montar en bicicleta.17,22 La actividad aerobia regular ayuda a mejorar la salud física y mental, atenuando a su vez el riesgo de mortalidad por obesidad. Algunas medidas recomendadas para personas que carecen de tiempo para realizar actividad física son: realización de múltiples episodios de 10 minutos de actividad física a lo largo del día, utilización de las escaleras en lugar del ascensor e implementación de escritorios de pie, algunos de los cuales vienen adaptados con cintas de correr. Es importante que los espacios de actividad física resulten agradables para los pacientes y que no representen incomodidad, por lo cual deben integrarse lentamente a la vida del individuo y se les debe orientar hacia la realización de alguna actividad que les resulte agradable, de lo contrario, no existirá adherencia. Por otro lado, se recomienda que en pacientes con alto grado de obesidad, primero se logre 


\section{CIENCIA\&SALUD}

una ligera disminución de peso mediante restricción calórica o ayudas farmacológicas, para posteriormente adicionar la actividad física 17,22

Disminuir el tiempo frente a las pantallas y otros comportamientos sedentarios es otra consideración importante para aumentar la actividad física, sin embargo, es importante tener en cuenta que la actividad física por sí sola produce una pérdida de peso mínima (solo $2 \%$ ) a seis meses si no se combina con restricción de calorías, por lo cual se anima a los participantes a implementar el ejercicio en su estilo de vida principalmente por su importancia en la salud y porque es fundamental para mantener la pérdida de peso a largo plazo, puesto que permite llevar una dieta poco menos restrictiva al incentivar la pérdida de un número mayor de calorías, por esto es muy importante que el paciente logre incorporarla a su estilo de vida y que le resulte agradable. Además, es importante mencionar que con el aumento de serotonina, adrenalina y demás hormonas relacionadas con la realización de la actividad física, puede contribuirse a la regulación de componentes ansiosos propios del paciente obeso, lo que a su vez, ayudaría a regular la ingesta de alimentos. 17,22

La adición de entrenamiento de fuerza al menos dos veces por semana, puede proteger contra la pérdida de músculo acompañada al proceso normal de envejecimiento.23

\section{Calidad de vida y salud mental}

Según escalas como la IWQOL, la pérdida de peso se asocia con marcadas mejoras en la función física y la autoestima, y mejoras clínicamente significativas en aspectos como la vida sexual, el trabajo y la angustia pública por el peso. Una mayor pérdida de peso generalmente se asocia con una mejora en la función psicosocial y satisfacción con la imagen corporal, sobre todo en personas que implementan actividad física recurrente en su estilo de vida. 17,24,25

La pérdida de peso moderada también se ha visto asociada con reducción de los síntomas de depresión, ansiedad, trastornos de la imagen corporal severa, trastornos de la alimentación y otras afecciones psiquiátricas que se encuentran en pacientes con obesidad, sin embargo, aunque se recomiendan terapias psicológicas en estos pacientes, no ha sido posible establecerlas como una intervención de primera línea para ellos, en la mayoría de los países, sobre todo en Latinoamérica, lo que dificulta el tratamiento integral de estos pacientes y disminuye las probabilidades de controlar esta patología, la cual constituye un problema de salud pública.17,24,25

\section{Terapia cognitivo conductual en el tratamiento de la obesidad}

La terapia cognitivo conductual es una terapia del habla en la que se trabaja con un experto en salud mental de forma estructurada con la finalidad de tomar conciencia sobre pensamientos o conductas imprecisas o negativas, con la finalidad de visualizar situaciones exigentes u objetivos con mayor claridad y de forma más eficaz.26

En el manejo integral de la obesidad, diversas guías han propuesto un acompañamiento psicológico necesario para que el paciente se adapte a su rutina de dieta y ejercicio, puesto que con el simple asesoramiento no basta, puesto que la obesidad es una enfermedad con componentes multifactoriales que afecta en diversos grados la psique del paciente.17

Diversos estudios han demostrado que la estrategia de tratamiento cognitivo conductual mejora la adherencia y disminuye la deserción del programa de reducción de peso, un ejemplo es el estudio realizado en 2014 con 115 adolescentes obesos entre 12 y 16 años que se dividieron en un grupo que recibió terapia y otro que solo recibió asesoramiento sobre un estilo de vida saludable. Los resultados de este estudio demostraron que el porcentaje de adolescentes con adherencia en el grupo de terapia fue de 73,7\% para la dieta y 61,4\% para el ejercicio, versus $41,4 \%$ y $19 \%$ respectivamente para quienes solo recibieron asesoramiento, por lo cual se concluyó que los pacientes del grupo de intervención exhibieron una disminución mayor y sostenida del peso corporal, el IMC y el porcentaje de grasa, demostrando que la terapia es efectiva.26 


\section{CIENCIA\&SALUD}

Otro ensayo realizado en pacientes obesos en los que se aplicó terapia cognitivo conductual junto con presoterapia por tres meses concluyó que los pacientes obesos a los que se les aplicó la intervención perdieron como mínimo $3 \mathrm{~kg}$ en menos de 3 meses y modificaron efectivamente sus hábitos de vida, por lo cual las medidas resultaron efectivas. 27 Estos hallazgos son similares a los encontrados en un estudio con pacientes que sufrían de obesidad mórbida y se habían sometido a cirugía bariátrica (CB) en un período inferior a dos años. En este estudio se encontró que los factores psicológicos representan un papel sumamente importante en los resultados de la CB, puesto que promueven una mayor estabilidad psicológica, lo que contribuye al mantenimiento del peso perdido puesto que los pacientes demostraron una menor preocupación por la comida y una mejor adaptación a nuevos hábitos de vida.28

Tabla 2. Contribuyentes de obesidad2,3

\begin{tabular}{cc}
\hline Intervención & Funcionamiento \\
\hline Programas comunitarios: & $\begin{array}{c}\text { Consiste en sesiones de tratamiento grupales que } \\
\text { permitirían dar terapia por un costo menos elevado } \\
\text { Efectos farmacológicos no deseados }\end{array}$ \\
\hline Programas comerciales: & $\begin{array}{c}\text { Servicios particulares que ofrecen sesiones } \\
\text { presenciales o en línea y paquetes de alimentación } \\
\text { grupal o individual basándose en las guías de } \\
\text { manejo de obesidad y problemas metabólicos. } \\
\text { El costo de estos suele ser elevado por lo que el } \\
\text { potencial beneficiario puede verse limitado a su } \\
\text { acceso }\end{array}$ \\
\hline Control de peso por teléfono: & Está pensado para personas que viven en áreas \\
& rurales o alejadas del centro de atención \\
\hline
\end{tabular}

Intervenciones para el control de peso entregadas Se basan en los métodos denominados eHealth (salud digitalmente electrónica) y mHealth (salud móvil), son asequibles y facilitan el establecimiento de objetivos, autocontrol, retroalimentación sobre el peso, la dieta y la actividad física.

Intervenciones basadas en internet:

Estos son más baratos y tienen mayor alcance potencial que la atención en persona. Su efectividad es mayor a largo plazo, puesto que a corto plazo el paciente experimenta una falta de responsabilidad al no haber una confrontación presencial, por lo cual la pérdida de peso disminuye en un $20 \%$ respecto a quien recibe atención en persona.30

Intervenciones a través de celulares

Las aplicaciones móviles tienen la capacidad de ofrecer contenido completo en cualquier momento y lugar, sin embargo, estas no son $100 \%$ personalizadas, lo que desmotiva al paciente y hace que la frecuencia de los inicios de sesión disminuya drásticamente con el tiempo17

Sin embargo, otros estudios demuestran que la terapia cognitivo conductual no es más efectiva que el consejo médico ni a corto ni a largo plazo, por lo que se proponen otro tipo de intervenciones, las cuales podrían ser de mejor acceso, como:29 


\section{CIENCIA\&SALUD}

Es importante aclarar que la efectividad de la terapia con respecto al consejo médico debe evaluarse teniendo en cuenta el número de terapias a las que el paciente tiene acceso, la accesibilidad a la misma, la oportunidad y la constancia, lo que puede dificultarse en países en los que el sistema de salud es débil y la consulta con especialistas no está disponible de forma oportuna para los pacientes. Además, se deben tener en cuenta condiciones individuales, como la edad.18

Un estudio realizado en el año 2020 por Baile, et. al., señala que la terapia cognitivo conductual sigue siendo el método de referencia para el tratamiento de la obesidad y que para que sea efectiva debe realizarse al menos una sesión semanal, con una duración de 16 a 24 semana, siendo recomendable que el período esté previamente definido para que puedan establecerse metas, las cuales, a su vez, deben ser flexibles de acuerdo con cada paciente y en caso de realizar terapias grupales debe hacerse un control estricto de los participantes sin permitir el ingreso tardío o la deserción de los integrantes.18

Respecto al grupo etario, en este mismo estudio mencionan que para los adolescentes puede ser efectiva la terapia de aceptación y compromiso; también podría utilizarse el tratamiento dentro de un programa multidisciplinar. En los adultos obesos, los programas multidisciplinares acompañados de terapia cognitivo conductual enfocada en la implementación de hábitos de vida saludable y mantenimiento de los mismos han resultado eficientes, pero se ha demostrado que en las mujeres se requiere de mayor acompañamiento psicológico. Por otro lado, los niños con esta patología requieren de terapia cognitivo conductual que pueda enseñarles a mejorar su estilo de vida de formas más dinámicas.18

\section{Farmacoterapia}

Las opciones farmacológicas actualmente disponibles son bastante limitadas y la mayoría están pensadas para enviar a pacientes con un IMC $>27 \mathrm{~kg} / \mathrm{m} 2$ con factores de riesgo asociados, o para aquellos con IMC >30. El tratamiento debe ser interrumpido a los 3 meses si ha perdido menos del 5\% del peso corporal.19

Estos tratamientos farmacológicos por sí solos suelen ser ineficaces a largo plazo tras su interrupción, por lo cual siempre deben articularse con el acompañamiento y educación del paciente para que logre adaptarse a un estilo de vida diferente, cambie su relación con su cuerpo y la manera en la que se alimenta, de lo contrario, toda medida terapéutica puede resultar ineficiente.

Tabla 3: Medicamentos en tratamiento de obesidad19

\begin{tabular}{|c|c|}
\hline Medicamento & Mecanismo de acción \\
\hline \multirow[t]{2}{*}{ Orlistat: } & $\begin{array}{l}\text { Consiste en sesiones de tratamiento grupales que permitirían dar terapia por un } \\
\text { costo menos elevado }\end{array}$ \\
\hline & Efectos farmacológicos no deseados \\
\hline Liraglutida: & $\begin{array}{l}\text { Inhibe irreversiblemente las lipasas pancreáticas, previniendo la absorción en } \\
\text { hasta } 32 \% \text { de ácidos grasos libres. Este conduce a una disminución del peso } \\
\text { corporal, colesterol y triglicéridos }\end{array}$ \\
\hline Naltrexona/bupropion: & $\begin{array}{l}\text { El primero es un opioide antagonista autorizado para el manejo de dependencia } \\
\text { a opioides y alcohol, mientras que el segundo es utilizado como ayudante para } \\
\text { dejar de fumar y actúa inhibiendo la captación de dopamina y noradrenalina. } \\
\text { Aunque no se conoce su efectividad se sabe que ambos conducen a la supresión } \\
\text { del apetito, puesto que reprimen los centros de hambre ubicados en el hipotálamo }\end{array}$ \\
\hline Lorcaserin: & Agonista de la serotonina que actúa de forma centralizada para suprimir el apetito. \\
\hline Fentermina/topiramato: & $\begin{array}{l}\text { El primero es un supresor del apetito de acción central y el segundo un } \\
\text { antiepiléptico que en combinación parecen inducir la pérdida de peso, por una } \\
\text { mayor utilización de energía }\end{array}$ \\
\hline Tratamiento quirúrgico: & Balón gástrico, cirugía bariátrica \\
\hline
\end{tabular}




\section{CONCLUSIÓN}

La obesidad constituye un problema de salud pública que afecta generalmente a los países más desarrollados, donde prevalecen las conductas sedentarias y donde los alimentos grasosos, ricos en grasas saturadas y ultra procesados, son abundantes y de fácil acceso a nivel monetario. Esta enfermedad, aunque está fuertemente asociada con el estilo de vida, también cuenta con componentes genéticos, sociales, psicológicos y metabólicos (endocrinológicos) que son determinantes a la hora de evaluar su curso y desarrollo, por lo cual, la implementación de un tratamiento integral es indispensable para poder combatirla, puesto que diversos estudios han demostrado que cuando se abarcan todas las esferas del paciente, haciendo énfasis en un acompañamiento para corregir conductas sociales y creencias psicológicas, se logra un mejor control de peso, sin efecto rebote. Por esto es de vital importancia implementar medidas de acompañamiento y educación a todo paciente obeso o con factores de riesgo para desarrollar obesidad, puesto que se ha visto que herramientas como la psicoterapia, terapia grupal, acompañamiento psicológico, entre otros, resultan indispensable para tratar de forma eficiente este problema de salud pública.

\section{Conflicto de interés}

No se declara ningún conflicto

\section{Declaración de financiamiento}

Este artículo no ha recibido financiamiento por ninguna entidad

\section{REFERENCIAS BIBLIOGRÁFICAS}

1) Ling C, Rönn T. Epigenetics in Human Obesity and Type 2 Diabetes. Cell Metab. 2019;29(5):102844. doi: 10.1016/j.cmet.2019.03.009.

2) Martínez JA, Milagro FI, Claycombe KJ, Schalinske KL. Epigenetics in adipose tissue, obesity, weight loss, and diabetes. Adv Nutr. 2014;5(1):71-81. doi: 10.3945/an.113.004705.

3) Park SS, Skaar DA, Jirtle RL, Hoyo C. Epigenetics, obesity and early-life cadmium or lead exposure. Epigenomics. 2017;9(1):57-75. doi: 10.2217/epi-2016-0047.

4) NCD Risk Factor Collaboration (NCD-RisC). Worldwide trends in body-mass index, underweight, overweight, and obesity from 1975 to 2016: a pooled analysis of 2416 population-based measurement studies in 128.9 million children, adolescents, and adults. Lancet. 2017;390(10113):2627-42. doi: 10.1016/ S0140-6736(17)32129-3.

5) Samblas M, Milagro FI, Martínez A. DNA methylation markers in obesity, metabolic syndrome, and weight loss. Epigenetics. 2019;14(5):421-44. doi: 10.1080/15592294.2019.1595297.

6) Ogden CL, Carroll MD, Kit BK, Flegal KM. Prevalence of childhood and adult obesity in the United States, 2011-2012. JAMA. 2014;311(8):806-14. doi: 10.1001/jama.2014.732.

7) Cawley J, Meyerhoefer C. The medical care costs of obesity: an instrumental variables approach. J Health Econ. 2012;31(1):219-30. doi: 10.1016/j.jhealeco.2011.10.003.

8) Casanello P, Krause B, Castro-Rodríguez J, Uauy R. Epigenética y obesidad. Rev. chil. pediatr. 2016;87(5):335-42. doi 10.1016/j.rchipe.2016.08.009. 


\section{CIENCIA\&SALUD}

9) Rosen ED, Kaestner KH, Natarajan R, Patti ME, Sallari R, Sander M, et al. Epigenetics and Epigenomics: Implications for Diabetes and Obesity. Diabetes. 2018;67(10):1923-1931. doi: 10.2337/ db18-0537.

10) Campión J, Milagro FI, Martínez JA. Individuality and epigenetics in obesity. Obes Rev. 2009;10(4):383-92. doi: 10.1111/j.1467-789X.2009.00595.x.

11) Robertson KD. DNA methylation and human disease. Nat Rev Genet. 2005;6(8):597-610. doi: $10.1038 / \mathrm{nrg} 1655$.

12) van Dijk SJ, Molloy PL, Varinli H, Morrison JL, Muhlhausler BS; Members of EpiSCOPE. Epigenetics and human obesity. Int J Obes (Lond). 2015;39(1):85-97. doi: 10.1038/ijo.2014.34

13) Ling C, Groop L. Epigenetics: a molecular link between environmental factors and type 2 diabetes. Diabetes. 2009;58(12):2718-25. doi: 10.2337/db09-1003.

14) Vickers $\mathrm{MH}$. Early life nutrition, epigenetics and programming of later life disease. Nutrients. 2014;6(6):2165-78. doi: 10.3390/nu6062165.

15) Lai CQ, Smith CE, Parnell LD, Lee YC, Corella D, Hopkins P, et al. Epigenomics and metabolomics reveal the mechanism of the APOA2-saturated fat intake interaction affecting obesity. Am J Clin Nutr. 2018;108(1):188-200. doi: 10.1093/ajcn/nqy081.

16) Stel J, Legler J. The Role of Epigenetics in the Latent Effects of Early Life Exposure to Obesogenic Endocrine Disrupting Chemicals. Endocrinology. 2015;156(10):3466-72. doi: 10.1210/en.2015-1434.

17) Wadden T, Tronieri J, Butryn M. Lifestyle Modification Approaches for the tratment of obesity in adults. Am Psychol. 2020; 75(2) 235-51 doi: 10.1037/amp0000517.

18) Baile J, Gonzales C, Paolo R, Rabito M. La intervención psicológica de la obesidad: desarrollo y perspectivas. 2020; 3 1-14 doi: 10.5093/cc2020a1

19) Ruban A, Stoenchev K, Ashrafian H, Teare J. Current treatments for obesity. Clinical Medicine. 2019; 19(3) 205-12

20) U. S. Department of Health and Human Services and U. S. Department of Agriculture. 20152020 Dietary Guidelines for Americans: 8th Edition. December 2015 Available at http:// health.gov/ dietaryguidelines/2015/guidelines/2015-2020

21) Franz MJ , VanWormer JJ, Crain AL et al. Weight-loss outcomes: a systematic review and meta-analysis of weight-loss clinical trials with a minimum 1-year follow-up. J Am Diet Assoc 2007 ; $107: 1755-67$.

22) Heymsfield SB, van Mierlo CA, van der Knaap HC, Heo M, Frier HI. Weight management using a meal replacement strategy: meta and pooling analysis from six studies. Int J Obes Relat Metab Disord $2003 ; 27: 537-49$.

23) Butryn ML, Webb V, Wadden TA. Behavioral treatment of obesity. Psychiatric Clinics of North America. 2011; 34(4), 841-59. 


\section{CIENCIA\&SALUD}

24) Powell KE, King AC, Buchner DM, Campbell WW, DiPietro L, Erickson KI, Whitt-Glover MC. The scientific foundation for the physical activity guidelines for Americans. Journal of Physical Activity \& Health. 2018; 2(17) 1-11.

25) Chao HL. Body image change in obese and overweight persons enrolled in weight loss intervention programs: a systematic review and meta-analysis. PloS one. 2015; 10(5)

26) Kolotkin RL, Norquist JM, Crosby RD, Suryawanshi S, Teixeira PJ, Heymsfield SB, Nguyen AM. One-year health-related quality of life outcomes in weight loss trial participants: Comparison of three measures. Health and Quality of Life Outcomes. 2009; 7(53).

27) Moran R, Mendoza E, Cumplido A, Simental LE, Rodriguez G, Sánchez GJ, et al. Cognitive behavioral treatment in the integral managment of obesity in adolescents. Rev Med Inst Mex Seguro Soc. 2014; 52(1) 68-73

28) Torres V, Castro AM, Matarán GA, Lara L, Aguilar M, Moreno C. Beneficios de la terapoa cognitivo conductual y la presoterapia en pacientes obesos. 2011; 26(5) 1018-24

29) Abilés V, Abilés J, Rodríguez S, Luna V, Martín F, Gándara N, Santaella MC. Efectividad de la terapia cognitivo-conductual en la pérdida de peso tras dos años de ciruhía bariátrica en pacientes con obesidad mórbida. 2013; 28(3) 1109-14

30) Bustos G, Moreno F, Calderín M, Martínez J, Martínez E, Arana C. Comparative study of medical advice and cognitive behavioral group therapy in the treatment of child-adolescent obesity. 1997; 47(2) 135-43

31) Harvey-Berino J, West D, Krukowski R, Prewitt E, VanBiervliet A, Ashikaga T, \& Skelly J. Internet delivered behavioral obesity treatment. Preventive Medicine. 2010, 51(2), 123-28. 